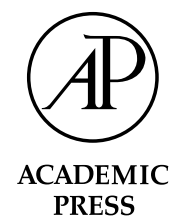

\title{
The contribution of EEG coherence to the investigation of language
}

\author{
Sabine Weiss ${ }^{\mathrm{a}, \mathrm{b}, *}$ and Horst M. Mueller ${ }^{\mathrm{b}}$ \\ ${ }^{a}$ Cognitive Neuroscience Group, Brain Research Institute, University of Vienna, Vienna, Austria \\ ${ }^{\mathrm{b}}$ Experimental Neurolinguistics Group - SFB 360, Faculty of Linguistics, University of Bielefeld, P.O. Box 100131, D-33502 Bielefeld, Germany
}

Accepted 7 February 2003

\begin{abstract}
The contribution of EEG coherence analysis to the investigation of cognition and, in particular, language processing is demonstrated with examples of recent EEG studies. The concept of EEG coherence analysis is explained, and its importance emphasized in the light of recent neurobiological findings on frequency-dependent synchrony as a code of information processing between nerve cell assemblies. Furthermore, EEG coherence studies on naturally spoken and written word and sentence processing are reviewed and experimental results are presented giving new insights into the occurrence of "transient functional language centers" within the brain.
\end{abstract}

(C) 2003 Elsevier Science (USA). All rights reserved.

Keywords: EEG coherence; Phase analysis; Frequency bands; Concrete; Abstract; Word processing; Verbal memory; Sentence processing; Spoken language; Review

\section{Why study EEG coherence during language processing?}

\subsection{Localized or distributed language function?}

Before the 19th century the common theory of brain function, which was termed "holism," assumed that the brain was not simply a structure made of discrete independent centers, each specializing in different functions, but must be looked upon as a single working unit. However, at the beginning of the 19th century scientists began to assign functions to certain neuronal structures and started to favor the theory of localization of function within the brain (Finger, 1994). The first localization of linguistic function to a specific region and hemisphere of the brain to become widely accepted is generally attributed to the French surgeon, Pierre Paul Broca (1824-1880), and the German neurologist, Carl Wernicke (1848-1905). In the 1960s the American neurologist, Norman Geschwind (1926-1984), refined Wernicke's model of language processing and this socalled Wernicke-Geschwind model still forms the basis

\footnotetext{
${ }^{*}$ Corresponding author. Fax: +49-521-106-6447.

E-mail address: sabine.weiss@univie.ac.at (S. Weiss).

URL: http://www.univie.ac.at/cognitiveneuroscience/.
}

of current investigations on normal and disturbed language function (Damasio \& Geschwind, 1984).

Although one cannot doubt the important roles of the well-known "language centers" in language processing, modern lesion and functional neuroimaging studies suggest that the classical Wernicke-Geschwind model, though useful for so many years, has to be extended. Language function is executed in both a localized and distributed manner. It may be true that certain components of language processing are carried out in particular brain locations (e.g., Binder, 1997), but mere activation of these locations does not allow intact language function. Different language operations are based on the dynamic mutual exchange of information between language relevant (not necessarily specific) locations (see also Mesulam, 1998). Thus, there is a need for hybrid neurophysiological models of language processing, which consider both localized and distributed patterns of information transfer during language processing to solve at least some open questions. Among them: Which brain structures specifically or necessarily participate in language processing and how are they organized to overcome requirements of different subcomponents of language processing? What does the pattern of interaction between participating neuronal 
structures look like and how is it coordinated? In particular, the later question is connected with studies on neuronal synchronization processes in the brain.

\subsection{Brain oscillations and language processing}

During the time-course of sentence comprehension different achievements of the language system, such as auditory perception, phonological, morpho-syntactic, semantic, pragmatic, and prosodic analyses have to be integrated in order to understand the meaning of the sentence and to initiate appropriate behavior. This is one example for the so-called binding problem, which has been theoretically addressed and neurophysiologically investigated in the past 20 years, mainly for visual object perception (e.g., Singer \& Gray, 1995; for review). The binding problem in cognitive neuroscience mainly deals with the way the brain integrates signals, separated in space and time, such that a unity is experienced. One of the better-known hypotheses proposing a neuronal code for integrated information processing is the temporal correlation hypothesis (Singer \& Gray, 1995). This hypothesis states that, under certain circumstances, neurons with similar feature properties can synchronize their discharges. Neuronal synchrony has been shown for adjacent neurons in the cat's and monkey's visual, auditory, motor, somatosensory and association cortices ("local-scale synchronization"; Abeles et al., 1993; Eckhorn et al., 1988; Eggermont, 1992; Gray, Koenig, Engel, \& Singer, 1989; Murthy \& Fetz, 1992) and between neuronal assemblies of distant brain regions, e.g., between somatosensory, motor, visual and parietal association cortices during a visuo-motoric task ("largescale synchronization"; Roelfsema, Engel, Koenig, \& Singer, 1997). Large-scale synchronization seems particularly important with respect to distributed neuronal assemblies, which have to be integrated during complex cognitive processing (Bressler \& Kelso, 2001; Varela, Lachaux, Rodriguez, \& Martinierie, 2001) and especially during language processing (for review Petsche \& Etlinger, 1998; Weiss \& Rappelsberger, 1996).

Large-scale neuronal synchronization and the nature of brain oscillations during cognitive information processing can be studied with EEG/MEG techniques. One approach to gain information on frequency band-related neuronal synchrony between different EEG/MEG signals is the computation of coherence. Studies on coherence range from intracortical recordings in animals via subcortical and subdural to scalp EEG/MEG recordings in normal humans and patients. In general, brain regions that are activated by cognitive operations show increased coherence ( $=$ neuronal cooperation or synchronization) within certain frequency bands, depending on the nature and difficulty of the task. It appears that each mental operation is accompanied by characteristic coherence patterns (Petsche \& Etlinger, 1998).

\section{What is EEG coherence?}

One method to quantitatively measure the linear dependency between two distant brain regions as expressed by their EEG activity is the calculation of coherence. Scalp recorded EEG coherence is a large-scale measure, which depicts dynamic functional interactions between electrode signals. High coherence between EEG signals recorded at different sites of the scalp hint at an increased functional interplay between the underlying neuronal networks.

The first application of coherence analysis to human EEG signals was not performed before the 1960s due to the lack of appropriate mathematical algorithms, computational power and computer software (Walter, 1968). With the increasing development of computerized techniques, a broader application of coherence analysis to EEG signals of healthy humans started in the 1970s with the first attempt to correlate cognitive functions with changes in coherence (e.g., French \& Beaumont, 1984, for critical review). However, a general acceptance and increasing interest in EEG coherence analysis as a method to monitor frequency dependent large-scale synchronization during human intact and disturbed information processing was not established before the 1990s, and the number of studies on EEG coherence and cognitive information processing in healthy humans has exponentially increased during the past four years. Detailed reviews on methodical aspects of EEG coherence analysis are given in Shaw (1984), Challis and Kitney (1991), Schack, Grieszbach, Arnold, and Bolten (1995), Nunez et al. (1997), Rappelsberger (1998); on clinical aspects in Leoncani and Comi (1999), Towle, Carder, Khorasani, and Lindberg (1999) and on cognitive aspects in French and Beaumont (1984) and Petsche and Etlinger (1998).

\subsection{Classical spectral analysis}

Mathematically, the coherence function is obtained by cross-spectral analysis, which is an essential part of EEG spectral analysis, since it enables us to quantify the relationships between different EEG signals. In short, the EEG is a complex signal with both stochastic (nonstationary) and deterministic (stationary) properties. In order to analyze different aspects of EEG signals, many commonly used methods are available, which can be roughly divided into two basic categories: parametric and non-parametric methods. Parametric models, such as autoregressive (AR) and autoregressive moving average (ARMA) filters or models (see below) assume, that the EEG is generated by a specific model (filtered noise), whereas non-parametric methods, such as conventional spectral analysis, do not assume a specific model for the generation of the EEG signal. The most commonly used method for EEG time series analysis is spectral analysis. 
In spectral analysis, EEG signals are transformed from the time domain into the frequency domain, which is typically performed by Fourier transform (FT). FT displays the properties of the data as a function of frequency and estimates a signal's frequency spectrum (power spectrum). This is justified by the fact that each time series can be represented as a sum of elementary sinusoidal frequency components. The multiplication of the Fourier transform of one signal with the complex conjugate of another signal yields the cross-power spectrum. The coherence function is obtained by the normalization of the cross-power spectrum and is basically written as magnitude-squared coherence. Coherence $(C)$ at a frequency $(f)$ for two signals $x$ and $y$ is derived from the cross-power spectrum $|S x y(f)|$ and the two corresponding autospectra, $\operatorname{Sxx}(f)$ and $S y y(f)$; $C^{2} x y(f)=|\operatorname{Sxy}(f)|^{2} / \operatorname{Sxx}(f) * \operatorname{Syy}(f)$. In practice, averaging techniques have to be applied to improve spectra estimation (Rappelsberger, 1998). Coherence values lie within a range from 0 to 1 whereby 0 means that corresponding frequency components of both signals are not correlated; 1 means frequency components of the signals are fully correlated with constant phase shifts, although they may show differences in amplitude.

In order to deal with certain constraints of correlation analysis of band-pass filtered signals or classical spectral analysis based on FT the use of sophisticated new approaches such as an ARMA model with time varying parameters to calculate coherence for small time steps seems promising (see Section 2.2). In addition, the calculation of phase relations to receive information on the direction and speed of information transfer may be applied (see Section 2.4). Furthermore, other parameters such as phase synchrony may be calculated, which avoids the influence of amplitude changes over single epochs (e.g., Lachaux, Rodriguez, Martinerie, \& Varela, 1999). Moreover, not only the investigation of synchronized activity within a single frequency range but also between different frequencies yields important new information on brain dynamics during cognitive processing (Schack et al., 2001; Schack, Vath, Petsche, Geissler, \& Moeller, 2002).

\subsection{Dynamic cross-spectral analysis by means of bivar- iate ARMA processes}

Classical spectral analysis based on FT relies on the stationarity of the EEG signal during the analysis epoch. Stationarity means that the statistical properties of the signal, such as mean, variance, and frequency content, do not vary with time. However, in most cases stationarity can only be assumed for short epochs. Classical FT allows one to monitor whether a certain rhythm appeared anywhere in the course of the epoch analyzed. If this information suffices to answer a certain experimental question non-stationarity can be ignored. How- ever, FT is then not informative on how frequency content changes with time inside a given time interval and is limited by poor frequency resolution for short data intervals. In particular, during cognitive processing subtle time-dependent changes in the oscillatory behavior of signals are often expected and have to be detected and monitored by advanced analysis methods. Owing to the non-stationarity of EEG signals, the use of non-linear methods or linear methods with parameters varying with time is recommended (Schack et al., 1995). Schack et al. developed an adaptive fitting algorithm of bivariate ARMA models with time-dependent parameters to estimate coherence and phase. This algorithm enables adaptation to structural changes in the signals and allows continuous investigation of spectral parameters with a high frequency resolution according to the dynamic changes of the signal. This parametric procedure has the capacity to produce smooth, high-resolution spectra even from short data sets. The dynamic behavior of coherence and phase, calculated by means of a bivariate adaptive ARMA model estimation with time-varying parameters, was extensively examined for word processing (Rappelsberger, Weiss, \& Schack, 1999; Schack, Rappelsberger, Anders, Weiss, \& Moeller, 2000; Schack et al., 2001; Schack, Rappelsberger, Weiss, \& Moeller, 1999b; Schack, Weiss, \& Rappelsberger, 1996; Weiss, Mueller, \& Rappelsberger, 1999) and other mental activities (e.g., Schack, Chen, Mescha, \& Witte, 1999a; Schack \& Krause, 1995).

\subsection{Interpretation of coherence}

The calculation of coherence provides an analytical tool by which signal content of two recordings can be monitored and quantified. Background noise (in the sense of uncorrelated activity of neuronal assemblies) may occur sporadically or continuously in one or both signals, new frequency components may add up into the signals and particular frequencies may change their amplitude. Moreover, phase between components in the two signals may alter over time. Therefore, coherence may also be interpreted as a measure for stability of phase between the same frequency components of two simultaneously recorded EEG signals. High coherence between two EEG signals means high cooperation and synchronization between underlying brain regions within a certain frequency band (Fig. 1).

For example, if high coherence exists between electrodes F3 and F7 in a certain time interval during language processing but not during rest or music processing, one may conclude that intense cooperation or neuronal synchronization within left frontal areas is important for language processing.

Since in general the EEG suffers the inverse problem, namely that localization of neuronal generators is weak, the former conclusion may be criticized. Nevertheless, 


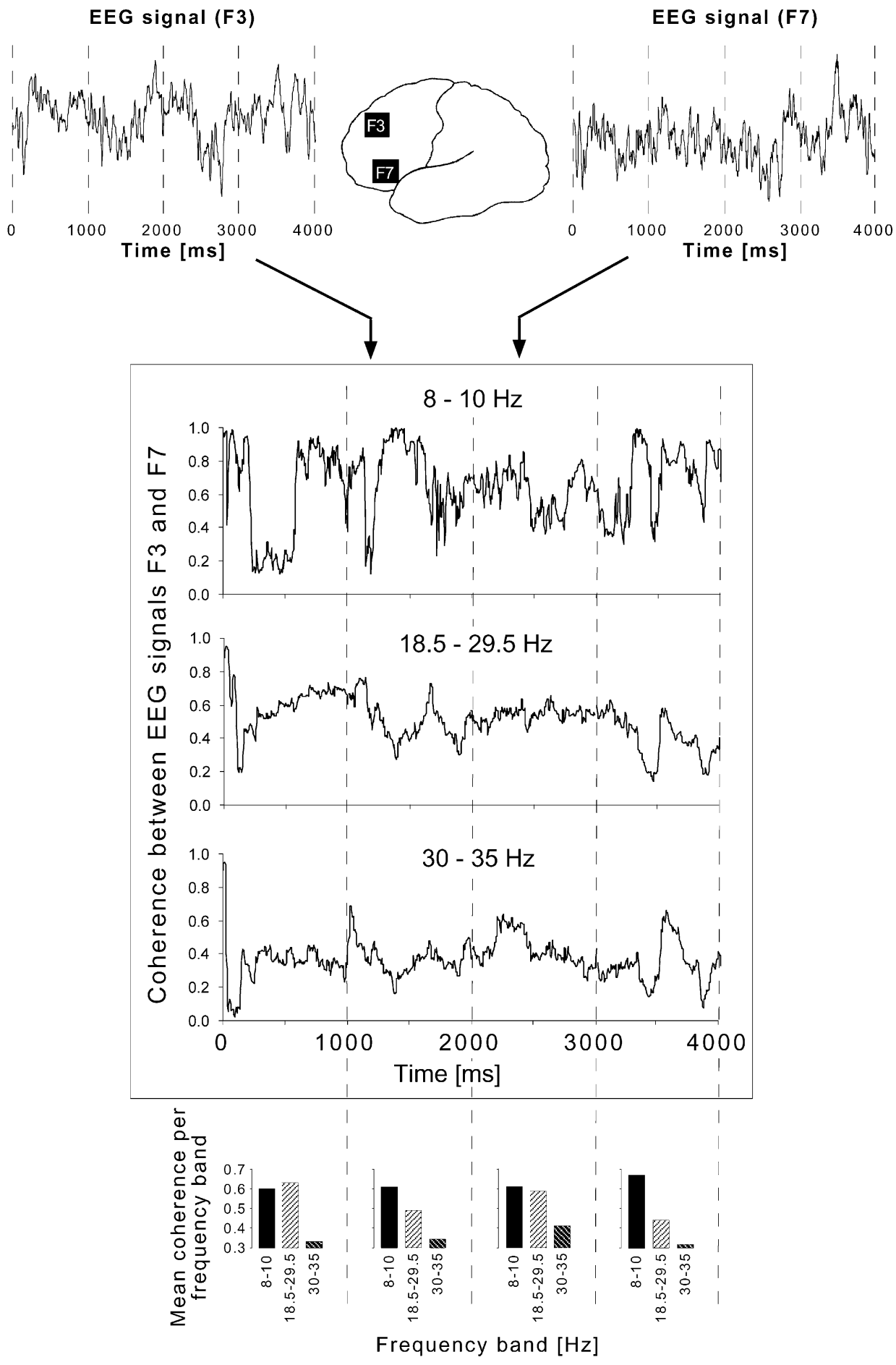

Fig. 1. An example for coherence analysis between two EEG signals. The EEG signals at F3 and F7 are recorded with scalp electrodes for four seconds (upper panel). Each frequency band illustrated shows specific coherence values dependent on the time interval investigated (see box). Whereas, for instance, mean coherence in the $8-10 \mathrm{~Hz}$ band does not change much across the four seconds, mean coherence in the $18.5-29.5 \mathrm{~Hz}$ band differs considerably (see bar histograms below).

there are some arguments which suggest that this criticism is misdirected. First, the EEG signal at a single electrode on the scalp consists of the summed electrical field potential from an area of $\approx 10 \mathrm{~cm}^{2}$ of the underlying cortex (e.g., von Stein, Rappelsberger, Sarnthein, \&
Petsche, 1999). This is the mean size of a cortical area and therefore it is plausible, that the electric activity at F3 and F7 stems primarily from the frontal cortex. However, this does not exclude another common neuronal source which influences this area. Therefore one 
may conclude that high coherence between F3 and F7 hints at an increased synchronization between the underlying frontal areas, although not knowing exactly where the original neuronal generators are located. Alternatively, it may be argued that coherence between F3 and F7 is just due to volume conduction into the two electrodes and therefore does not reflect true synchronization between underlying neuronal groups. Volume conduction would lead to an amplitude (or power) increase at both electrode sites. Therefore, if amplitude does not increase at both electrode sites or even decreases, which frequently is the case, volume conduction can be excluded. Furthermore, the distance between electrodes $(\approx 6 \mathrm{~cm})$ does not suggest volume conduction. Further discussion on the interpretation of coherence can be found in Petsche and Etlinger (1998).

\subsection{Computation of phase relations}

Additionally, further knowledge on the dynamic behavior of synchronization processes assessed by the measurement of direction (time delay) and speed of information transfer between brain areas is significant. Information on these parameters can be obtained by the calculation of phase relations between oscillatory components of two signals. Phase and coherence are, mathematically, closely connected spectral parameters, and interpretation of phase is only sensible with sufficiently high coherence values. The cross-phase spectrum provides information on the time relationships between two EEG signals as a function of frequency. It may be estimated by the inverse tangens (arctan) of the imaginary $(\mathrm{Im})$ part divided by the real $(\mathrm{Re})$ part of the crosspower spectrum; $\varphi(f)=\arctan (\operatorname{Im} S x y(f)) /(\operatorname{Re} S x y$ $(f)$ ). Phase displacement may be converted into time displacement. The sign of phase demonstrates the direction of information transfer per frequency, while the amount of phase is a measure for the speed of information transfer (Rappelsberger et al., 1999; Schack et al., 1999b). A measured time delay indicates that the signal recorded at one site precedes or lags behind the signal recorded at another site. Typically, coherence and phase estimations are based on FT (Rappelsberger et al., 1999), as described above, but can also be obtained by the application of parametric methods such as the twodimensional approach of adaptive filtering to estimate coherence and phase continuously (Schack et al., 1999b).

\subsection{Relationship between ERPs and EEG coherence}

Up to now, the great majority of EEG-studies on language processing have employed the analysis of event-related potentials (ERP), which are time-locked to a particular event and which are extracted from background EEG by signal-averaging techniques. ERP studies have revealed important results on the precise temporal processing of language stimuli (for review Brown \& Hagoort, 1999; Kutas, 1997), although certain drawbacks have to be considered. First, ERPs consist of phase-locked activity, which is quantified by averaging procedures, whereas non-phase-locked activity is attenuated. Thus, the ERP is only a rough estimate and a first approximation of the whole brain response (Basar, 1998). Interactive brain systems are more likely to reveal changes through the rates and duration of their activities than through amplitude fluctuations as analyzed in the ERP-method. Secondly, the EEG is a bioelectric signal, which consists of rhythmic activity in several frequency ranges. Activity in different frequency ranges is correlated with different subcomponents of cognitive tasks, which may be processed in parallel within overlapping networks. For instance, the perception and semantic portions of a task seem to be reflected within different frequency bands of the EEG (e.g., Basar, 1998; Weiss \& Rappelsberger, 1996, 1998). Without specific analysis ERPs do not provide information on activity within certain frequency bands. Moreover, coherence analysis is able to monitor dynamic large-scale synchronization between activities at electrodes placed at different scalp locations, which complements the findings obtained by ERP analysis.

\section{EEG coherence and language processing}

Although, the application of coherence analysis to EEG data during language processing has proven itself a promising tool for investigating rhythmic, large-scale properties of EEG signals accompanying linguistic function, relatively few studies on this topic have been performed (for review Petsche \& Etlinger, 1998; von Stein et al., 1999; Weiss et al., 1999; Weiss \& Rappelsberger, 1996, 1998). One reason for this may be that until recently there was a lack of appropriate computer power to perform coherence analysis and to efficiently handle coherence data. Other reasons may be the "Zeitgeist," which only recently began to recognize the importance of such a measure and the strong tradition of using ERP-analysis for language processing.

\subsection{Clinical studies on dyslexia}

In general, due to their main topics, EEG coherence studies can be divided into clinical studies and studies with healthy subjects. In this section we shall merely be concerned with coherence studies on patients with language disorders as the main pathological symptom. To the best of our knowledge, apart from the extensive studies on dyslexic patients there are no studies on EEG coherence and specific language disorders. Dyslexia, a developmental disorder of reading, is commonly treated 
as a heterogeneous syndrome, as are the results of the EEG coherence studies. One of the earliest studies was made by Sklar, Hanley, and Simmons (1972) in which 12 dyslexic children and 13 healthy children had to perform various mental tasks including reading word lists and text. Sklar et al. found higher coherence within hemispheres (intrahemispheric) and lower coherence between hemispheres (interhemispheric) in dyslexics than in normals during text processing. These findings were supported by Leisman and Ashkenazi (1980) in 20 dyslexic patients. However, during rest Shiota, Koeda, and Takeshita (2000) reported both increased intra- and interhemispheric coherence in dyslexic children compared to healthy subjects. Furthermore, Marosi et al. (1995) found a frequency-dependent effect on coherence differences between children with poor reading/writing abilities compared with children with good reading/ writing abilities, with the former showing higher coherence in the $\delta, \theta$, and $\beta$ bands and lower coherence in the $\alpha$ bands during rest. Nagarajan et al. (1999) examined evoked MEG coherence responses in the auditory cortex of adults with poor and good reading abilities. Adults with poor reading abilities showed lower average $\beta$ and $\gamma$ $(20-60 \mathrm{~Hz})$ coherence compared with controls. These heterogenous results may be a reflection of differences in methodology or groups of patients with different clinical syndromes. Particularly, early studies on EEG coherence suffered from technical problems extensively discussed in French and Beaumont (1984). Taking these findings together, general tendencies for a reduced coherence can be found in dyslexic patients compared to healthy subjects during language processing.

\subsection{Language processing in healthy adults}

\subsubsection{Word processing}

The very first studies, which tried to demonstrate the influence of word processing on EEG coherence, dealt with word fluency tasks, word generation and priming effects. However, they reported somewhat heterogeneous findings that did not seem to have inspired their authors to continue their work on these topics. Therefore, only single studies were reported, which sometimes suffered from methodological problems and were not continued or extended (Beaumont, Mayes, \& Rugg, 1978; Sheppard \& Boyer, 1990; Tucker, Dawson, Roth, \& Penland, 1985). Other studies on EEG coherence and word processing dealt with word category differences and are addressed in the following sections.

\subsubsection{Effects of category differences on word perception.} In the cognitive neuroscience of language one of the main questions raised concerns the cortical representation of the mental lexicon. Up to now it is not known whether the division into different word types, such as verbs and nouns or concrete and abstract nouns, cor- relates with different neurophysiological processing in the brain. The subdivision into different word types can be traced back at least 2000 years, and is based on theoretical findings in speech philosophy and linguistics. During the last two decades, neuropsychological and brain imaging studies on patients with brain lesions have been concerned with the search for the neurobiological basis of lexical processing. Selective category-specific impairments of grammatical word types, such as nouns versus verbs, have been reported (e.g., Caramazza \& Hillis, 1991). Moreover, category-specific impairments exist for abstract versus concrete nouns (e.g., Warrington \& Shallice, 1984), subgroups of concrete nouns, such as proper names and common nouns (e.g., Semenza \& Zettin, 1989) and even for subtypes of common nouns such as persons, tools or animals (e.g., Damasio, Grabowski, Tranel, Hichwa, \& Damasio, 1996). These findings were supported by brain imaging studies with healthy participants, which reported on different functional processes and/or distinct functional networks involved in processing different word types. In correspondence with the findings in patients, healthy subjects also show different brain activation for nouns and verbs (e.g., Pulvermueller, Preissl, Lutzenberger, \& Birbaumer, 1996, 2001; Warburton et al., 1996), concrete and abstract nouns (e.g., Kiehl et al., 1999; Weiss \& Rappelsberger, 1996; West \& Holcomb, 2000), proper names and common nouns (e.g., Mueller \& Kutas, 1996) and even subgroups of verbs (Pulvermueller et al., 2001; Weiss, Berghoff, Rappelsberger, \& Mueller, 2001a).

To summarize, some findings tend to support the existence of the representation of traditional linguistic word types within the brain. However, neurophysiological evidence indicates that the subdivision into linguistic categories should be more differentiated. For example, imageability may characterize a verb better than its grammatical affiliation to the class of verbs (Weiss et al., 2001a) that also contributes to its neuronal representation (Kellenbach, Wijers, Hovius, Mulder, \& Mulder, 2002). In the following, some of our own experiments on EEG coherence and language processing, which deal with word category processing are shown.

First experiments were performed on the perception of different word categories without any specific task, to minimize the participation of additional cognitive processes. We were interested to learn: (1) whether common and/or specific coherence networks exist for each of the word types investigated, and (2) whether specific EEG frequency bands selectively reflect these effects.

In a first step, we tested if there were coherence networks common to the perception of all word types investigated (verbs, abstract nouns, and concrete nouns) and whether they occur both for the auditory and the visual modality of stimulus presentation. These coherence networks may reflect global processes, which are common to processing of single words independently of 
their syntactic or semantic affiliation, thus allowing them to be interpreted as coherence networks typical for word perception. These coherence networks are supposed to mediate attentional and mnemonic processes, which, in part, should be the same for the perception of different word types. The EEG of 25 healthy female students was recorded during either auditory or visual perception of 75 verbs, 75 concrete nouns, 75 abstract nouns, and 140 distractors randomly presented. Words were controlled for various psycholinguistic criteria (e.g., concreteness/ abstractness, imageability, meaningfulness, articulatory length, word frequency, and number of syllables). A detailed description of psycholinguistic criteria used in a comparable experiment was reported in Weiss and Rappelsberger (1998). Participants had to perceive the stimuli and listen to them carefully. EEG was recorded with 19 electrodes according to the 10/20 system against averaged signals of both ear lobes, which is the most suitable reference for computation of coherence (Essl \& Rappelsberger, 1998). Classical spectral analysis based on FT of $1 \mathrm{~s}$ epochs containing the word presentation was performed. Coherence between all possible electrode combinations was computed for the different word types and for the resting condition. For reasons of data reduction coherence was calculated for six selected frequency bands: $\delta$ (delta; $1-4 \mathrm{~Hz}) \theta$ (theta; $5-7 \mathrm{~Hz}$ ), $\alpha-1$ (alpha-1; 8-10 Hz), $\alpha-2$ (alpha-2; 11-12 Hz), $\beta-1$ (beta-1; $13-18 \mathrm{~Hz}$ ), and $\beta-2$ (beta-2; $19-31 \mathrm{~Hz}$ ). For the evaluation of significant differences between chosen parameters, paired Wilcoxon-tests (two-tailed) were applied. The rank sums obtained were converted to error probabilities and presented in topographic probability maps (see Figs. 2-5). On account of the many parallel statistical tests the results presented in those maps have only exploratory character but they can be used as statistical filters. A more detailed description of the procedures can be found in Rappelsberger and Petsche (1998) and in Rappelsberger (1998).

Fig. 2 demonstrates probability maps of coherence changes during word perception in comparison to the resting condition. The first column shows coherence changes, which are common to all auditorily presented word types whereas the second column shows coherence changes, which are common to all visually presented word types. The third column illustrates those "language-specific" coherence changes, which are common to both auditorily and visually presented word types.

Interestingly, main coherence changes, which also tend to be common for all word categories when considered separately, are found within low frequency bands $(1-10 \mathrm{~Hz})$ whereas almost no common coherence changes are displayed in the higher frequency bands $(11-31 \mathrm{~Hz})$. Previously, the $\delta$ band has been correlated with attention (Harmony, 1999) and $\theta$ with memory (e.g., Klimesch, 1999). Therefore, the patterns of coherence changes common to word perception are as- sumed to be related to basic processes, such as attention, memory and semantics in terms of meaning construction.

The question remains which role higher frequencies may play during word perception. Possibly, they could reflect syntactic differences between word types (e.g., verbs vs. nouns) and semantic processes in terms of categorization (abstract vs. concrete nouns). In a further step, we directly compared certain word types with each other to get information on specific differences between word types. Fig. 3 presents coherence differences between concrete nouns and verbs (first column) and between concrete and abstract nouns (second column) for higher frequency bands $(11-31 \mathrm{~Hz})$.

For the perception of concrete and abstract nouns the most striking coherence differences are found in the $\beta-1$ band $(13-18 \mathrm{~Hz})$. Concrete nouns display two main networks of higher coherence, a small one at left frontal electrodes and a larger one at posterior electrodes and between left frontal and right posterior electrodes. Obviously, cooperation between hemispheres is intensified during the perception of concrete nouns. Higher $\beta-1$ band coherence has also been described whilst memorizing concrete nouns, although the topography of coherence was somewhat different (Weiss \& Rappelsberger, 1996). Concrete and abstract nouns are represented within the brain as different functional networks (on top of considerable overlap) based on inherent semantic properties of each of the two word categories. Higher coherence for concrete nouns can be explained by the multimodal representation of concrete nouns (Weiss et al., 1999). Frequency band specific results were also found in a study of von Stein et al. (1999). Twentythree participants had to perceive objects as pictures, as spoken and as written words. The authors found increased $\beta-1$ coherence $(13-18 \mathrm{~Hz})$ between temporal and parietal electrodes to occur during the presentation of all three modalities. They suggested that synchronization between left hemispheric temporal and parietal cortices within the $\beta-1$ band occurred during semantic integration of objects. Similar findings on medium frequency range coherence were described by Schack et al. (1999a), who recorded the EEG of 10 participants during the Stroop test. They found higher $\beta-1$ coherence $(13-20 \mathrm{~Hz})$ both within and between the left frontal and left parietal electrodes for the incongruent case of color naming (word "red" written in blue ink). Right hemispheric coherence increased for the congruent case in comparison to the incongruent case.

The first column of Fig. 3 shows coherence differences between concrete noun and verb processing, which are mainly reflected by $\beta-1$ and $\beta-2$ frequency bands, with nouns particularly showing higher frontal coherence. At a first glance, this suggests different functional cooperation networks for nouns and verbs probably predominating at frontal sites. However, in a further study on 


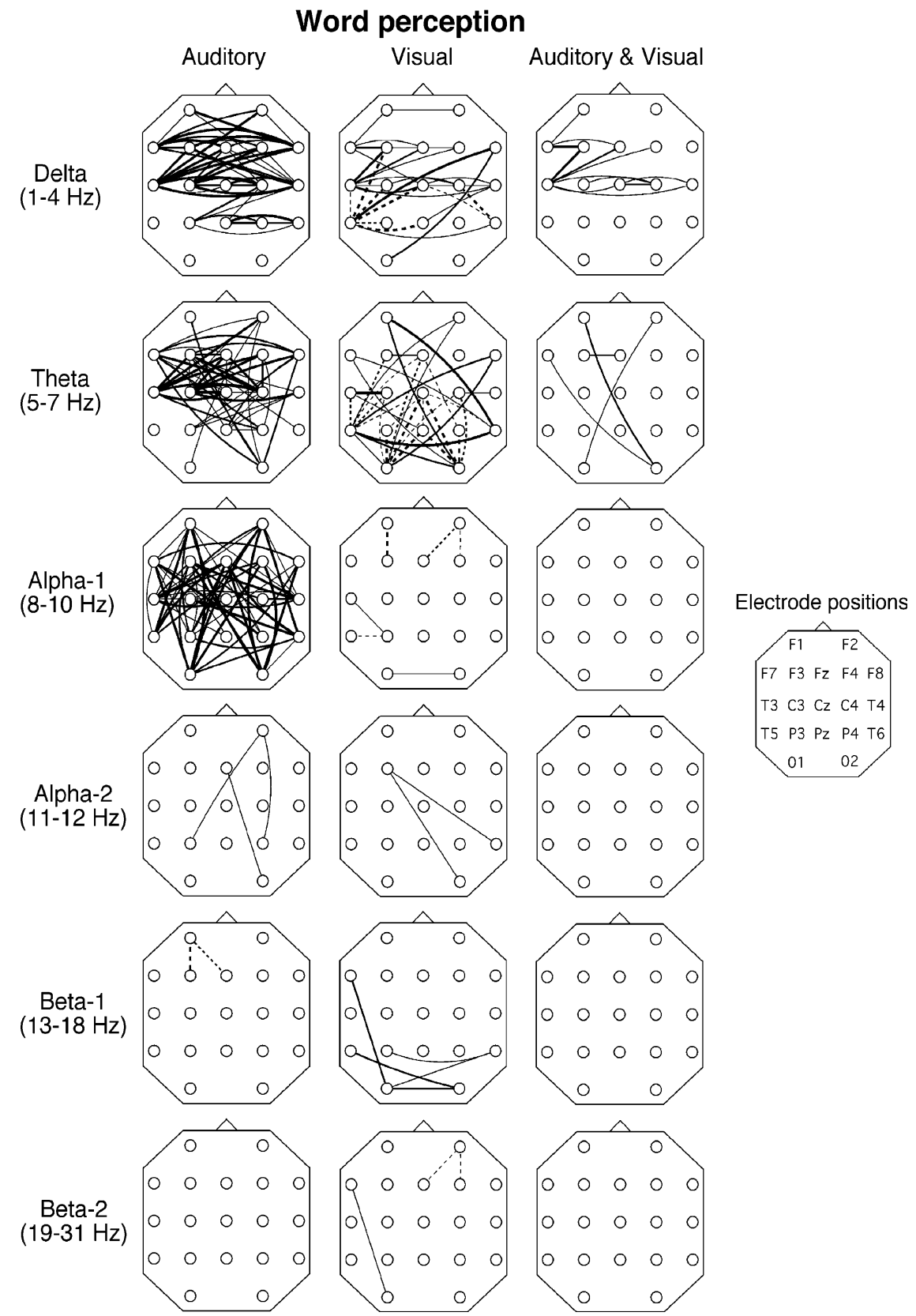

Fig. 2. Coherence changes common to abstract nouns, concrete nouns and verbs compared with a resting condition. There are coherence changes common to all auditorily presented word types (first column), to all visually presented word types (second column) and to both auditorily and visually presented word types (third column). Full lines indicate coherence increase and dashed lines indicate coherence decrease during word perception compared with the resting condition. Error probabilities are mapped onto schematic brain maps as connecting lines between the electrodes involved. The thickest line relates to an error probability of $p \leqslant .01$, the other lines to error probabilities of $p \leqslant .02$ and $p \leqslant .05$, respectively. Basically, low frequencies $(1-10 \mathrm{~Hz})$ reflect processes common to all word types whereas high frequencies $(11-31 \mathrm{~Hz})$ show almost no coherence changes common to all word types.

the perception of different subgroups of verbs it was shown that this assumption had to be expanded (Weiss et al., 2001a; Weiss, Mueller, King, Kutas, \& Rappelsberger, 2001b) and that results depend heavily on the types of verbs used. 52 participants had to rate 75 German verbs whether they belonged to concrete or abstract, high imagery or low imagery and motor- or non-motor-related subgroups of verbs. Verbs were matched for psycholinguistic criteria, which were described in a comparable experiment reported in Weiss and Rappelsberger (1998). The EEG of 23 participants was recorded during the random presentation of visually and auditorily presented verbs separated by 185 distractors. During perception of the stimuli, EEG was recorded at 19 electrodes according to the 10/20 system against averaged signals from both ear lobes. Analyses 


\section{Nouns minus verbs}

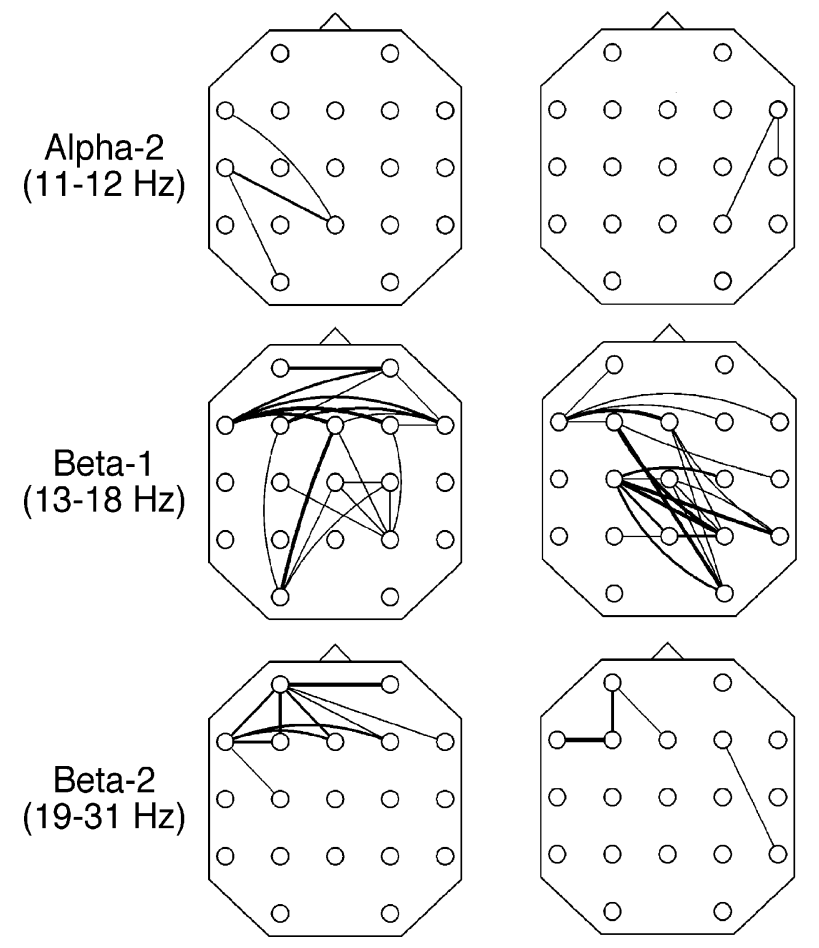

Fig. 3. Coherence differences between auditorily presented words in the frequency range from 11 to $31 \mathrm{~Hz}$. Concrete nouns lead to very few coherence values lower than the other word types, therefore only differences with higher coherence for concrete nouns are shown. Significance of coherence changes correlates with the thickness of the lines between two electrodes (see legend Fig. 2).

were similar to those in the previous experiment. Results indicated massive coherence differences between each subgroup of verbs. The most obvious difference was found between high imagery (e.g., to lie) and low imagery verbs (e.g., to name). Fig. 4 demonstrates left and right hemispheric coherence differences for high and low imagery verbs compared with the resting condition in the $\beta-2$ band.

High imagery verbs show increased left and right hemispheric coherence, especially between long distance frontal and posterior electrodes. The integration of visual association areas during processing of high imagery verbs seems plausible, since visual imagery is performed by the same neuronal structures, which also support visual processing and perception (Farah, 1989). These results also support recent findings that even verbs referring to actions executed with the legs (e.g., to kick) and face-related verbs (e.g., to speak) can be separated by the EEG (Pulvermueller et al., 2001).

In the same experiment on word perception reported above 40 German proper names and 40 common nouns were auditorily presented in randomized order with 120 distractors (Weiss, Mueller, \& Rappelsberger, 1998a).
Statistical analysis was based on ANOVAs and post hoc paired Wilcoxon-tests (two-tailed). The $\alpha-2$ band (11$12 \mathrm{~Hz}$ ) showed a significant interaction between word type and hemisphere. Whereas names elicited higher coherence in the right posterior hemisphere, common nouns elicited higher coherence between left posterior electrodes (Fig. 5). These findings strongly support the proposed special role of names (Mueller \& Kutas, 1996) within the category of concrete nouns.

To summarize, word perception elicits various patterns of coherence changes within both low and high frequencies of the EEG. Lower frequencies $(1-10 \mathrm{~Hz})$ tend to reflect non-specific components of word processing such as sensory, attentional, mnemonic and basic semantic parts of the task, whereas higher frequencies $(11-31 \mathrm{~Hz}$ and possibly higher) reflect specific coherence patterns, which differ depending on the word class/category investigated. However, no specific single higher frequency band seems to be exclusively responsible for "word type differences". Each of the higher frequency bands may reflect most significant and typical coherence differences depending on the kind of words compared. It would seem that neuronal synchronization patterns during word processing are based on a very complex relationship between participating oscillations in various frequencies. Current evidence does not allow a definite statement on the role of both lower and different higher frequencies besides the fact that higher frequencies seem predominantly to reflect cognitive-linguistic word type differences.

\subsubsection{Category types during verbal memory encoding.} An additional major topic of our research on EEG coherence and language concerns the investigation of memory processes during language comprehension. Findings described above were supported by experiments dealing with the memorization of lists of either concrete or abstract nouns (Weiss \& Rappelsberger, 1996, 1998). Nouns were either auditorily or visually presented and had to be recalled immediately after the presentation of each list. Results indicated $\alpha-1$ to be mainly engaged in the sensory processing of stimuli. In the $\beta-1$ band auditorily presented concrete nouns elicited higher coherence between distributed brain regions than abstract nouns. Weiss and Rappelsberger (1996) hypothesized that due to the multimodal nature of concrete nouns more distributed brain areas are functionally synchronized and, thus cooperate, whereas more local coherence networks accompany abstract nouns. Concrete and abstract nouns are based on different "main nodal points" and connections that may link separate brain regions. Common coherence patterns were predominately found in lower but not in higher frequency bands. Results were supported by a study of Volf and Razumnikova (1999), who applied coherence analysis to 30 subjects whilst they memorized 


\section{High imagery verbs}

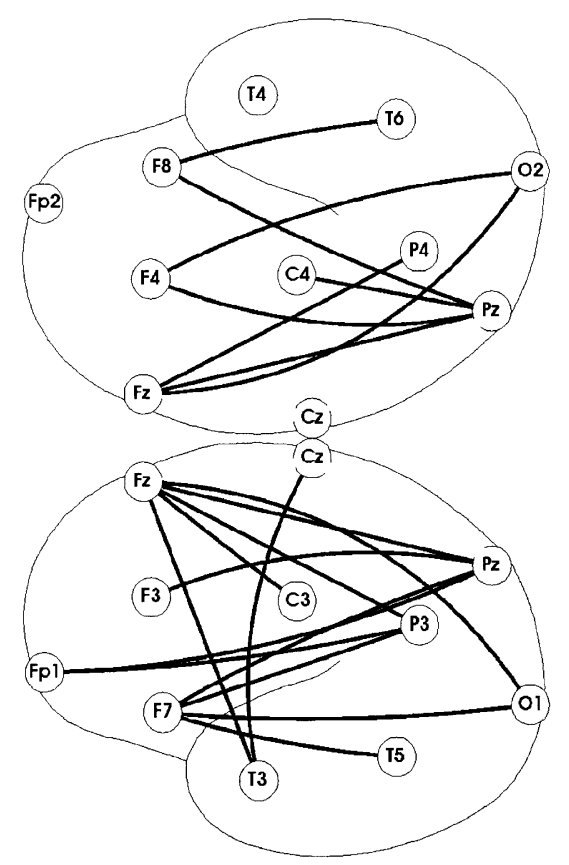

Low imagery verbs

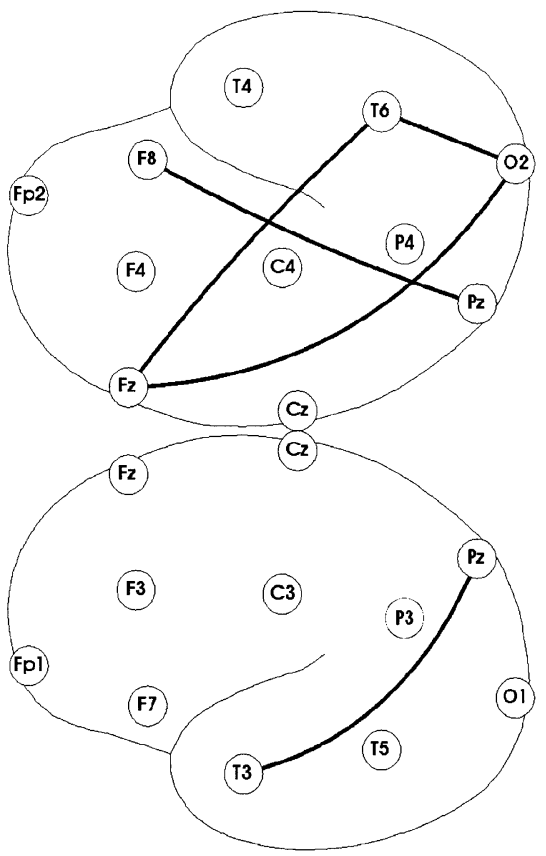

Fig. 4. Intrahemispheric coherence differences between auditorily presented high and low imagery verbs compared with the resting condition in the $\beta-2$ band $(19-31 \mathrm{~Hz})$. Error probabilities $(p \leqslant .05)$ are mapped onto schematic maps of the unfolded left and right hemisphere. High imagery verbs show increased coherence, especially between frontal and posterior electrodes.

\section{Names minus nouns}

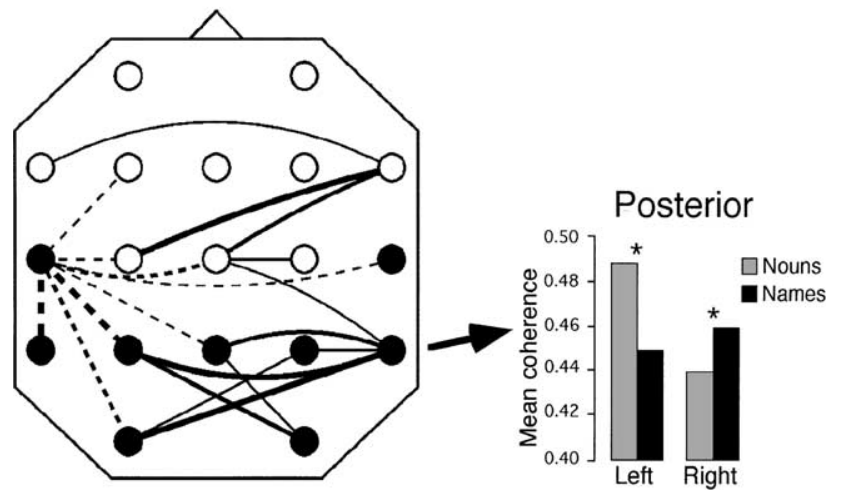

Fig. 5. Map of coherence differences between proper names and common nouns in the $\alpha-2$ band (11-12 Hz). (Significance of differences: see Fig. 2.) Full lines indicate higher coherence and dashed lines indicate lower coherence for proper names. Mean significant coherence differences for names and nouns at posterior electrodes (filled circles) revealed by paired $t$ tests $(*=2 p \leqslant .05)$.

dichotically presented lists of concrete nouns. Besides frequency band specific results, mainly coherence increase was found compared to a resting condition.

In the first coherence study focusing on categorial word processing Rugg and Venables (1980) reported differences in intertemporal coherence between learning of high imagery and low imagery words. They found that participants, who showed a small difference in the recall of concrete and abstract nouns, had large differences in interhemispheric $\alpha(7.8-12.1 \mathrm{~Hz})$ coherence and small differences in interhemispheric $\theta(3.9-7.4 \mathrm{~Hz})$ coherence. Thus, memory performance was correlated with contrary effects in adjacent frequency bands, which led them to speculate on different functional roles of these frequency bands in mnemonic and linguistic processing.

We performed experiments in order to compare coherence during the encoding of subsequently recalled and non-recalled concrete and abstract nouns presented either auditorily or visually (Weiss, Mueller, \& Rappelsberger, 2000; Weiss \& Rappelsberger, 2000). The major result of these studies was that during word encoding recalled nouns elicited significantly higher mean coherence than non-recalled nouns. Enhanced coherence occurred independently of the modality of stimulus presentation and the type of verbal material used. However, the topography of this overall higher coherence was slightly different for word types such as concrete and abstract nouns. This means that words, which were likely to be recalled, were correlated with an increase of synchronized activity between various brain regions. In particular, synchronization between frontal and posterior sites and between both hemispheres seemed to be necessary for the successful encoding of words. This study provided the first hint that increased synchronization of electric activity between distant brain regions enables efficient verbal memory encoding and further may allow us to decide whether a word is likely to be recalled or not. 
In addition to these results, we were interested in getting information on the direction of information transfer within these coherence networks and also on the propagation speed during word processing. Therefore, in addition to coherence, phase relations were calculated during the $1 \mathrm{~s}$ epoch of word memorizing (Rappelsberger et al., 1999). As mentioned in Section 2 coherence describes the amount of information transfer, whereas cross-phase enables determination of the direction of information transfer. Previous results on concrete and abstract noun processing in the $\alpha-1$ and $\beta-1$ bands were confirmed with phase analysis (Fig. 6). Arrows pointing from leading to trailing electrodes indicate direction of information transfer.
In $\alpha-1$ almost identical patterns of the direction of information transfer are obtained for concrete and abstract nouns. In contrast, in the $\beta-1$ band clear differences appear between concrete and abstract word processing with regard to transfer between occipital and frontal $(\mathrm{O} 1-\mathrm{F} 3, \mathrm{O} 2-\mathrm{F} 4)$ and occipital and fronto-polar (O1-Fp1, O2-Fp2) leads. Thus, not only the coherence patterns shown before but also phase relations are different for concrete and abstract nouns in the $\beta-1$ band.

However, with the FT only information on coherence patterns averaged over the whole second containing word presentation is obtained. Due to this lack of high temporal resolution, we were interested in also monitoring dynamic coherence changes during word

\section{Concrete nouns}

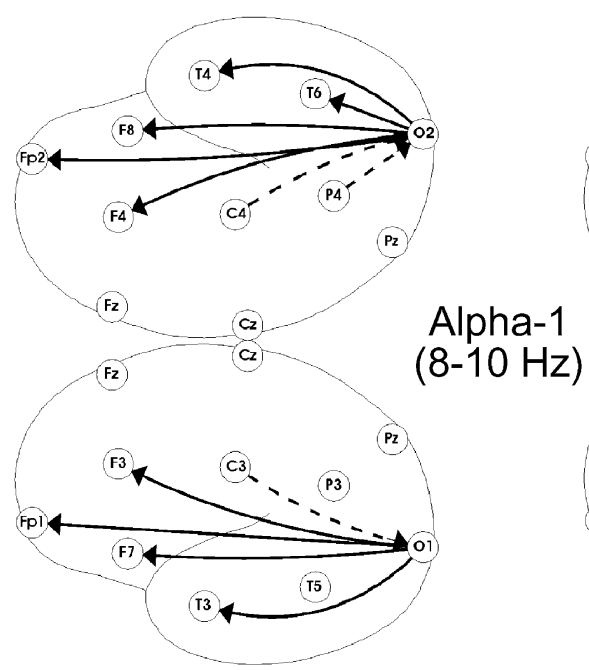

Abstract nouns

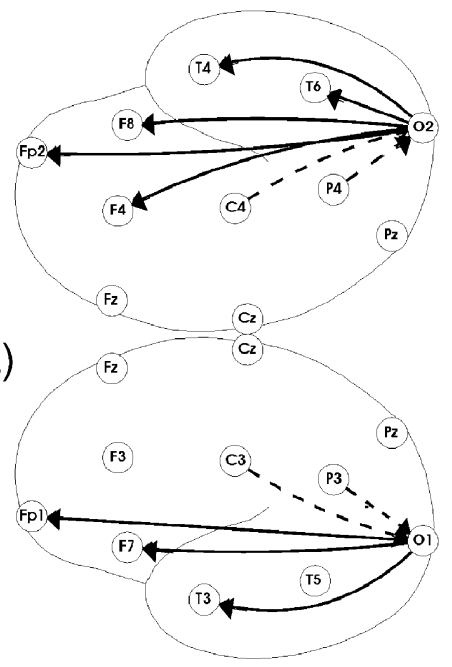

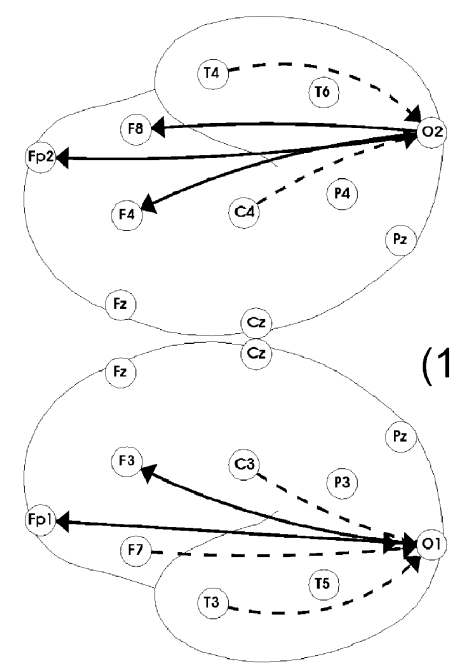

Beta-1

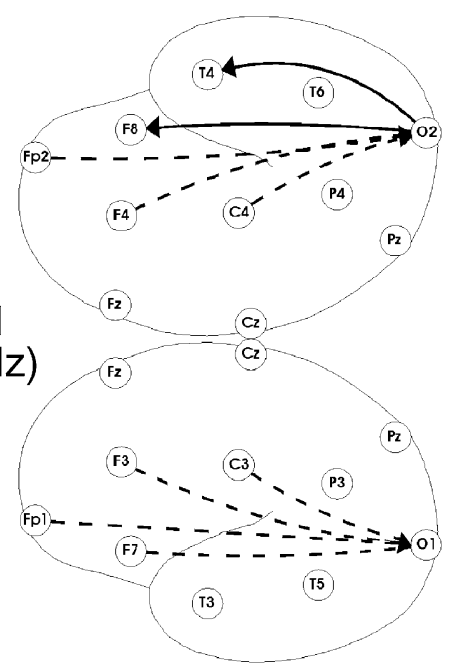

Fig. 6. Examples of mean phase relations of 25 participants between $\mathrm{O} 1 / \mathrm{O} 2$ and all other intrahemispheric electrode positions during memorization of visually presented nouns. Direction of information transfer is indicated by arrows and goes from leading to trailing electrode positions. Solid lines denote $\mathrm{O} 1 / \mathrm{O} 2$ as leading electrodes, dashed lines as trailing electrodes. The absolute time delays range from about 0 up to $17 \mathrm{~ms}$. Time relations exceeding $1 \mathrm{~ms}$ absolute are presented. Whereas almost no difference between the memorization of concrete and abstract nouns is found in the $\alpha-1$ band, the $\beta-1$ band clearly correlates with the task. 
processing. Data from the last experiment were used to apply an adaptive fit of a bivariate autoregressive moving average (ARMA) model (see Section 2), which allows monitoring of dynamic coherence changes during word processing within time windows in the millisecond range (e.g., Schack et al., 1996). This ARMA approach with time varying parameters was used to compute instantaneous coherence and phase values. Coherence changed considerably every $200 \mathrm{~ms}$ during word processing, thus reflecting short dynamic neuronal synchronization patterns. The computation of phase relations during visual presentation of abstract nouns indicated an information transfer from visual to frontal association areas within the first $400 \mathrm{~ms}$. After $400 \mathrm{~ms}$ the direction of information transfer changed and varied subsequently (Schack et al., 1999b). In addition, concrete and abstract words tended to differ in their processing speed within the brain. Concrete nouns had longer mean delays, which indicated that propagation of information while memorizing concrete nouns was slower (Schack, Weiss, \& Rappelsberger, 2003). Slower activation of more distributed networks can explain this. These results provide initial reasons to think that, in addition to conventional coherence analysis, phase estimation may yield valuable new insights into the physiology of word processing. With the application of the ARMA approach with time varying parameters, essential new information on dynamic coherence and phase changes during word processing was obtained.

\subsubsection{Text and sentence processing}

Neurophysiological studies on single word processing provide data on certain aspects of language processing. However, text and sentence processing have to be studied as well in order to get information on the neurobiological basis of the full set of mechanisms underlying language comprehension and production. Unfortunately, most EEG-studies focused on visual word-by-word recognition rather than on auditory text and sentence comprehension.

In a pilot study on text processing by Petsche, Etlinger, and Filz (1993) EEG coherence was determined for three professional, non-bilingual interpreters when interpreting from their native language into foreign languages and vice versa. Beyond interindividual differences, participants showed an increased involvement of the left temporal regions in the higher $\beta$ band (24$32 \mathrm{~Hz}$ ). Fewer EEG changes were found when the message was not understood. Increased right hemispheric participation was found when interpreting into the foreign language rather than into the native language. The authors described coherence changes accumulating at certain electrodes ("nodal points"), which are supposed to have functional significance for the task. For instance the electrode T3 seemed to be such an important nodal point in language interpreting. In another experiment
Petsche (1996) examined the EEG of 38 participants who had to construct a story incorporating 10 words they had previously learned. During this verbal creative act increased coherence between frontopolar and occipital electrode sites, indicating a stronger involvement of long cortico-cortical fiber systems, was observed.

No coherence studies on sentence processing have been published up to now either. The processing of sentences requires a complex scenario analysis via language, depending on various phonological, syntactic and semantic processes in time. Temporary representations in turn require some form of working memory (Caplan \& Waters, 1999; Mueller, King, \& Kutas, 1997a). In the following, results of two experiments on the processing of auditorily presented German and English sentences are shown.

3.2.2.1. Semantic violation of German sentences. We performed a pilot study on the processing of auditorily presented German sentences (Weiss, Rappelsberger, Schack, \& Mueller, 2003). In order to be able to compare results to existing ERP data on sentence processing we used a very well-known paradigm, which has been thoroughly studied in the literature, namely semantic violation. As has been known for 20 years the eventrelated potential to a semantically incongruous word within a sentence is characterized by a negative-going wave between 200 and $600 \mathrm{~ms}$ peaking around $400 \mathrm{~ms}$, the so-called N400 (Kutas \& Hillyard, 1980).

In the present study, 100 sentences were presented to 50 subjects in order to assess the cloze probability of their final words. Participants completed the sentences with what they thought were the most appropriate final words. The results of this pilot experiment were taken into account when selecting the final sentence material for the EEG experiment. During the EEG experiment 21 participants were asked to listen to auditorily presented sentences. 31 incongruous, 31 congruous and 27 distractor sentences were presented in a randomized order. Sentences were simple (Subject-Verb-Object) sentences and were presented with a stimulus onset asynchrony of $6 \mathrm{~s}$. Participants were instructed to listen attentively to the sentences in order to comprehend their meaning. The EEG was recorded with 19 scalp electrodes according to the 10/20 system against an average ear lobe reference, band-pass filtered $(0.3$ to $35 \mathrm{~Hz})$ and digitally sampled at $256 \mathrm{~Hz}$. In order to investigate general EEG coherence characteristics of sentence processing, coherence based on classical FT was calculated for 4 s EEG epochs after each sentence onset. No significant coherence difference was found for sentence comprehension compared to a pseudo-speech signal in the $\alpha-1(8-10 \mathrm{~Hz})$ band whereas in the $\beta-1$ band $(13-18 \mathrm{~Hz})$ sentence processing exhibited higher coherence, mainly at frontal and fronto-central electrodes (Mueller, Weiss, \& Rappelsberger, 1997b). On the basis of these results it was 
proposed that the $\alpha-1$ band reflects processes of primary acoustic analysis (which should not differ for the two conditions) whereas the $\beta-1$ band reflects cognitive analysis during German sentence processing.

Further, we were interested in the temporal dynamics of coherence while processing congruous and incongruous sentences. Therefore, coherence was computed based on an adaptive fit of an ARMA model (Schack et al., 1995) $1000 \mathrm{~ms}$ before and $1000 \mathrm{~ms}$ from onset of their final words. Time-frequency matrices for coherence were calculated for the electrode pair $\mathrm{Pz}-\mathrm{P} 4$. This electrode pair was selected since Pz commonly shows a very strong N400 effect in ERP analysis. The time-frequency information of coherence allows frequencies to be determined, where coherence changes correlate with the time evolution of the cognitive process and which discriminate between both sentence types. Fig. 7 shows time-frequency matrices of coherence for the electrode pair $\mathrm{Pz}-\mathrm{P} 4$ for congruous as well as incongruous sentences for a single participant.

The most prominent coherence differences for the congruous and incongruous final words were found between 300 and $500 \mathrm{~ms}$ after word onset for frequencies around $30 \mathrm{~Hz}$. Therefore, coherence at $30 \mathrm{~Hz}$ was statistically compared for congruous and incongruous sentences within three relevant time intervals. Paired $t$ tests were performed for the time intervals from $300 \mathrm{~ms}$ before word onset up to word onset, from word onset up to $300 \mathrm{~ms}$ and from $300 \mathrm{~ms}$ up to $600 \mathrm{~ms}$ after word onset. Coherence between 300 and $600 \mathrm{~ms}$ after word onset was significantly different for the two sentence types $(2 p \leqslant$ $0.05)$. This time interval also showed the expected $\mathrm{N} 400$ difference for congruous and incongruous sentences in the ERP, peaking around $376 \mathrm{~ms}$. Fig. 8 demonstrates the course of average coherence at $30 \mathrm{~Hz}$ for congruous and incongruous sentence processing for a single participant.

Within the time interval from 300 up to $600 \mathrm{~ms}$ after word onset coherence was significantly higher for congruous compared to incongruous words. These preliminary data suggest that higher coherence at $30 \mathrm{~Hz}$ for congruous words may be correlated with semantic integration processing (Weiss et al., 2003). However, further data are necessary to support these findings.

\subsubsection{Subject-subject and subject-object English rela-} tive clauses. This study deals with EEG coherence

\section{Sentence processing}

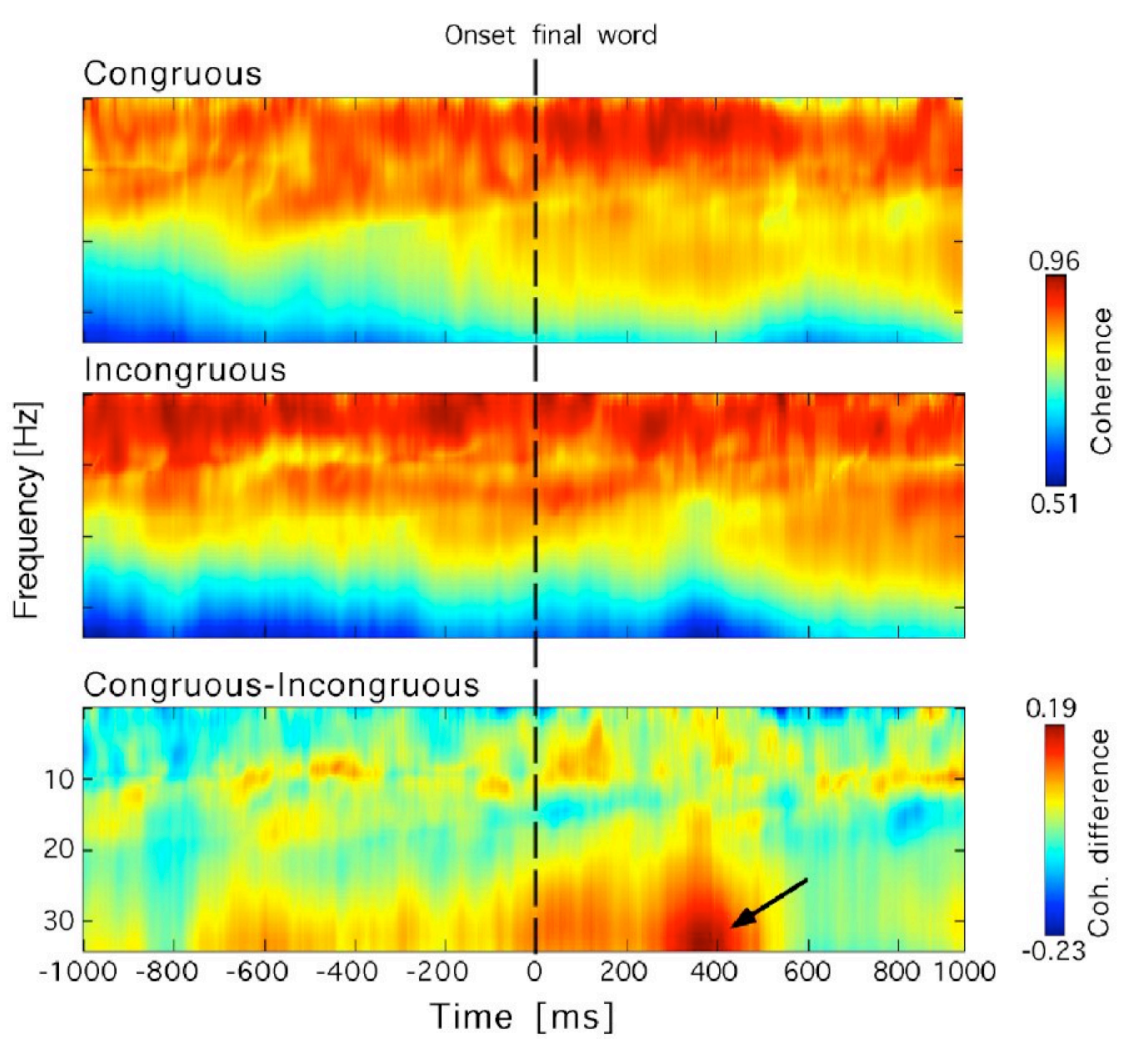

Fig. 7. Time-frequency matrices of averaged coherence for 31 congruous and 31 incongruous sentences (upper and middle panel) and the difference between them (lower panel). Matrices demonstrate time-frequency dependent differences of coherence for the electrode pair Pz-P4 for a single participant. In the difference map dark areas denote higher coherence for congruous sentences, particularly around $30 \mathrm{~Hz}$ during the final word (arrow). 
Gamma coherence $(30 \mathrm{~Hz})$ during sentence processing

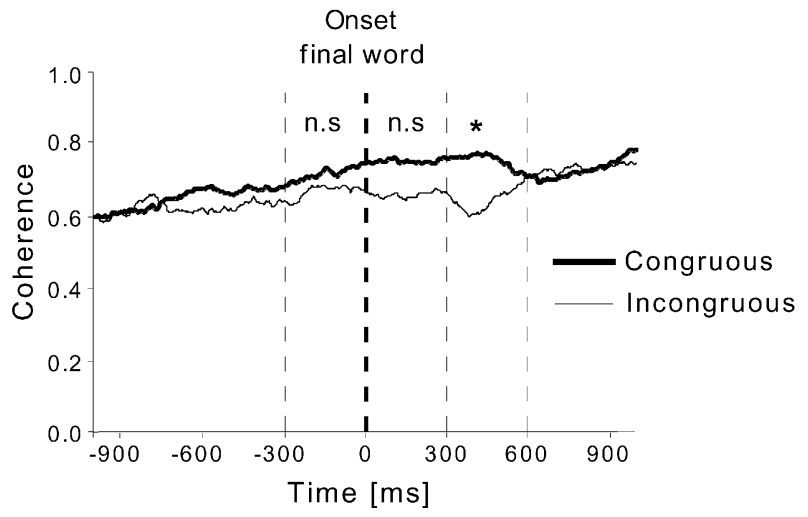

Fig. 8. Time course of mean coherence for the electrode pair Pz-P4 during processing congruous (thick line) and incongruous (thin line) sentences in a single participant. Significant differences are found in the time interval between 300 and $600 \mathrm{~ms}$ after onset of the final word $(*=2 p \leqslant .05)$.

analysis while subjects process English relative clauses (Weiss et al., 2001a, 2001b). Coherence analysis was applied to the data reported in Mueller et al. (1997a) in order to obtain new results in the frequency domain. The experimental paradigm used in this study is a very well known, thoroughly studied paradigm and therefore allows conclusions on the relationship of results obtained with coherence analysis to results obtained with other methods such as ERP or fMRI.
In this study two different types of relative clauses were used: in half of the sentences the subject of the main clause was also the subject of the relative clause (SS-sentences), while in the other half it served as the object of the relative clause (SO-sentences) (for examples, see Fig. 9, upper part). Generally, comprehension of SO-sentences is more difficult for the listener due to, among other things, greater demands on working memory. Studies with almost identical sentence material reported that $\mathrm{SO}$-sentences elicit more comprehension errors and slower reading times at and just following the end of the relative clause (King \& Just, 1991). ERPs to visually presented SS- and SO-sentences were different at the beginning of and after the relative clause, with higher left frontal negativities for the SO-sentences (King \& Kutas, 1995). Comparable ERP-results were found for auditorily presented SS- and SO sentences. However, effects were more widespread and the right hemisphere showed more pronounced differences $(\mathrm{Mu}-$ eller et al., 1997a). An fMRI-study has also found right hemispheric areas to be more active during visual processing of more difficult SO-sentences (Just, Carpenter, Keller, Eddy, \& Thulborn, 1996).

In the present study, EEG was obtained from 17 scalp sites as 24 participants listened to normally spoken English relative clauses. The critical material consisted of 72 sentences each containing either subject-subject (SS) or subject-object (SO) relative clauses. A detailed description of the experiment is given in Mueller et al. (1997a). Sentences were divided into five different peri-

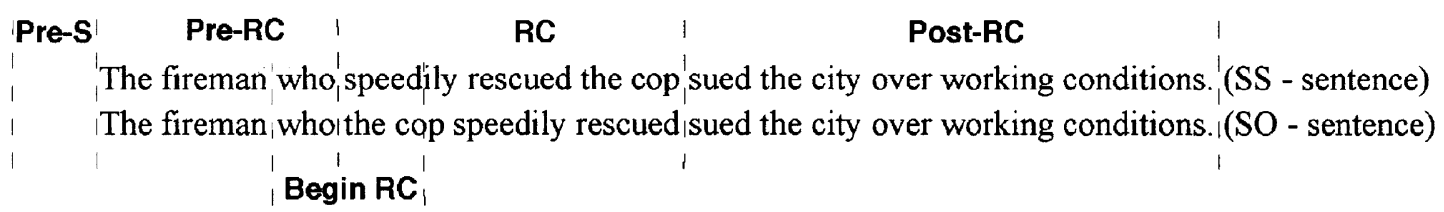

\section{Course of coherence during sentence processing}
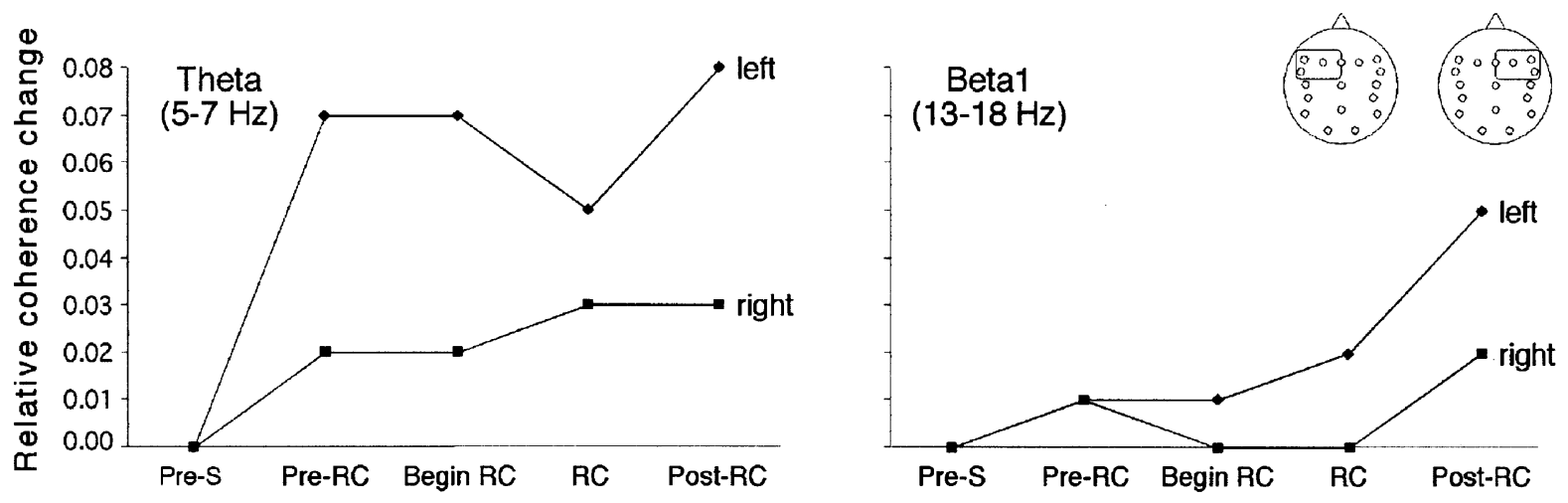

Sentence periods

Fig. 9. Upper part: Examples of a single sentence once as subject-subject (SS) and once as subject-object (SO) relative. Lower part: Course of mean coherence at left and right frontal electrodes (indicated on the head schemes) for selected sentence periods for 24 participants. Coherence in the pre-S interval served as baseline. Sentence processing leads to higher coherence at left hemispheric electrodes. 
ods: (1) period before sentence onset (pre-S), (2) period before relative clause onset (pre-RC), (3) beginning of the relative clause (begin-RC), (4) relative clause (RC) and (5) period after relative clause (post-RC) (Fig. 9, upper part). FT of artifact-free $1 \mathrm{~s}$ epochs of these predefined sentence periods was made. Finally, coherence was calculated for the $\theta(5-7 \mathrm{~Hz})$ and $\beta-1(13-18 \mathrm{~Hz})$ frequency bands.

At first, the general course of coherence during sentence processing irrespective of sentence type was studied. Mean coherence at left and right frontal sites was calculated for the selected sentence periods, coherence in the pre-S period served as baseline (Fig. 9, lower part).

Coherence increases at sentence onset and is more pronounced at left frontal sites than for right frontal sites for both frequency bands investigated, with the highest coherence in the post-RC. In particular, $\theta$ coherence increase in the course of sentence processing may be correlated with increased demand on working memory during processing of complex sentences.

In a further step, the difference in coherence between SO- and SS-sentences was compared within different sentence periods. The most striking difference between these sentence types was found for the coherence between left and right frontal sites and is demonstrated in Fig. 10.

SO-sentences show significantly higher coherence in the post-relative clause within the $\theta$ band. Within the $\beta-1$ band SO-sentences show higher coherence both at the beginning of the relative clause and in the post-relative clause. As mentioned above, ERP-results of these EEG data revealed higher frontal and central negativities for SO-relatives due to increased working memory load at the beginning of the $\mathrm{RC}$ and in the post-relative clause. Modulation of $\theta$ activity in humans is been commonly correlated with episodic and working memory (e.g., Klimesch, 1999; Sarnthein, Petsche, Rappelsberger, Shaw, \& von Stein, 1998) and $\theta$ power tends to increase in the course of sentence processing (Bastiaansen, van Berkum, \& Hagoort, 2002). Thus, we expected $\theta$ band coherence to differ between SO- and SS-relative clauses as soon as working memory load differs (at the beginning of the RC). However, only $\beta-1$ band coherence was significantly different in this time interval. At least four explanations can be given with respect to these findings. First, coherence is different from power measures in that it reflects large-scale synchronization whereas the later reflects local synchronization processes (see also Section 2.3). As a consequence, although $\theta$ power may reflect working memory processes (Bastiaansen et al., 2002) $\theta$ coherence does not necessarily have to. A second possibility is that $\theta$ is related to working memory and $\beta-1$ activity may correlate with the activation of a separate parsing buffer similar to that proposed by Caplan and Waters (1999). This means, that at the beginning of the relative clause the load of the parsing buffer significantly differs between the two sentence types whereas the load of both types of working memory does not significantly differ before the post-relative clause. A third possibility is that the $\beta-1$ band reflects working memory and $\theta$ is correlated with increased processing. Although there is no support from literature for this interpretation, possibly changes in $\beta$ band coherence reflect the activation of syntactic working memory whereas the $\theta$ band is influenced by increased task demand in the post-relative clause. Another more trivial explanation is that the ERP-results discussed above were most prominent in good comprehenders. We have not examined this factor in our study, which may also provide a reason that the $\theta$ coherence difference for SO- and SS-sentences did not

\section{SO- minus SS sentences}
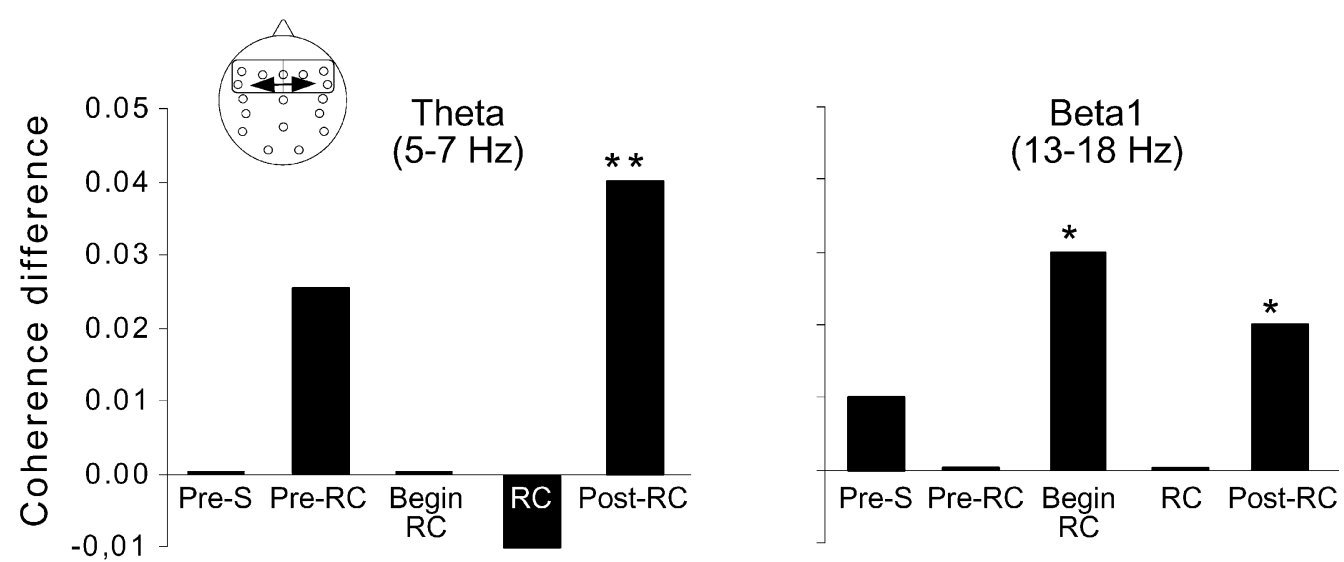

Fig. 10. Coherence difference between SO- and SS-sentences. Upright bars denote higher coherence for SO-sentences between left and right frontal sites (as indicated on the head scheme). Post hoc $t$ tests revealed significantly higher coherence for the SO-sentences in the $\theta$ band in the post-RC. The $\beta-1$ band coherence was higher for SO-sentences both at the beginning of the relative clause (begin $\mathrm{RC}$ ) and in the post-RC ( $*=p \leqslant .05$, $* *=p \leqslant .01)$ 
reached significance. At present, these questions have to remain open and further data are needed to clarify them. Nevertheless, some conclusions can be drawn concerning EEG coherence accompanying sentence processing (Weiss et al., 2001a, 2001b). Mean coherence increase during parsing of sentences is clearly higher within the left hemisphere. SS- and SO-relatives show different cooperation networks in $\theta$ and $\beta$ frequency bands, which possibly can be attributed to different aspects of sentence processing such as working memory and parsing. Moreover, results yield new insights into the relationship of EEG coherence to ERP analysis.

\section{Conclusion}

Computation of coherence between bioelectrical signals can be applied to measure the relationship between EEG signals recorded during cognitive function. Coherence reflects the degree of information flow between groups of neurons generating these EEG signals. Thus, coherence gives insights into the way functional networks cooperate with each other during various cognitive processes.

Summarizing findings in literature and our own findings, some general statements on the nature and meaning of EEG coherence accompanying language processing can be made. High coherence correlates with long-lasting negativities in the ERP and is often found during increased task complexity and efficient information processing, whereas low coherence is often found in pathological conditions. Creative language tasks are accompanied by an increase of long-range coherence and worse comprehension is correlated with coherence decrease. Depending on task demand either coherence networks within each hemisphere or between both hemispheres are elicited. During processing of complex language stimuli increased right hemispheric participation is found (Petsche \& Etlinger, 1998; Weiss et al., 2000). The relation between intra- and interhemispheric cooperation (coherence) is also influenced by the gender of the participants. Women seem to have higher interhemispheric and right hemispheric coherence whilst memorizing verbal stimuli (Volf \& Razumnikova, 1999). Coherence is also able to differentiate between the quality of performance of individuals and groups (Weiss, Cromecek, \& Rappelsberger, 1998b).

Furthermore, coherence is a frequency-dependent measure, and patterns of coherence networks tend to differ between frequencies. The meaning of coherence networks may be interpreted differently depending on the frequency band investigated, since different components of a cognitive task are presumably processed via different frequencies (Basar, 1998; Klimesch, 1999; Weiss \& Rappelsberger, 2000). According to Basar (1998), each complex and integrative brain function such as language processing elicits multiple oscillations (in $\delta, \theta, \alpha, \beta$, and $\gamma$ ranges) and is characterized by a superposition and participation of different frequencies. Thus, it is not possible to assign a single function to a given type of oscillatory activity.

During linguistic information processing our own studies point at different roles of high and low frequency-synchronization (e.g., Weiss \& Rappelsberger, 1996, 1998, 2000). The $\theta$ frequency band (around $3-7 \mathrm{~Hz}$; originates as a result of cortico-hypothalamic interaction) seems correlated with language-related mnemonic processes, and $\theta$ coherence increases if task demands increase and more efficient information processing is required. The $\alpha(8-12 \mathrm{~Hz}$; generated mainly but not exclusively by reverberating propagation of nerve impulses via cortico-thalamic connections) is probably important for sensory and, in the higher range, also for semantic processing. The $\beta(13-30 \mathrm{~Hz})$ and $\gamma$ $(>30 \mathrm{~Hz})$ (both presumably generated inside the cortex) seem to be correlated with more complex linguistic subprocesses such as syntax or semantics. In addition, in our studies we found specific coherence patterns within different frequencies (higher than $11 \mathrm{~Hz}$ ), and these patterns were different for certain word types, such as concrete and abstract nouns, high-imagery and lowimagery verbs, common nouns and proper names. High coherence apparently correlates with the increasingly multimodal features of certain word types. In addition, phase relations during word processing indicated direction of information and propagation speed of information transfer, which proved an important new parameter for studying cognitive processes. Coherence can also reflect operations during sentence processing and shows specific behavior to various aspects of sentence processing in different frequency ranges. At high frequencies coherence may be correlated with semantic integration and parsing processes.

These results argue that EEG-coherence analysis is an important tool for studying high-level cognitive processes, such as language processing. This method supports a somewhat different view on brain function during language processing in so far as the actual process of language comprehension and production is not correlated with location but with interaction. Particularly, coherence between distant electrodes challenges the localistic view that language function can be mostly attributed to definite circumscribed "language centers". Results of EEG coherence studies demonstrate "transient functional language networks", which sometimes are of a very short duration $(200 \mathrm{~ms})$. The typical appearance of these "transient functional language networks" depends on the kind of verbal stimuli, the task and on the individual experience of persons performing the task (see also Petsche \& Etlinger, 1998). Large-scale information transfer via frequency coding is possibly one of the mechanisms which facilitate parallel pro- 
cessing within the brain, since a single signal may contain different aspects of information within various frequency ranges. This may be one reason for the high speed of information processing. Furthermore, the assumption that "transient functional language centers" exist may partly explain the spontaneous recovery from acquired aphasic disturbances. After a certain period of neuronal reorganization missing functions can be taken over by "new functional language centers" based on the function of existing neuronal ensembles.

Further systematic studies on EEG coherence and language will elucidate and clarify the meaning and interpretation of previous findings. The combined application of different analysis methods (e.g., ERP analysis) and other neurophysiological techniques (e.g., eventrelated fMRI) seems particularly promising in this context. Cortical cooperation in patients with aphasia or related syndromes may be studied in the course of remission to establish which patterns of coherence are correlated with clinical symptoms and with returning function. Among many other successfully applied neurophysiological methods, the description of functional networks during language processes using coherence analysis provides a small but important piece of the mosaic on our way to understanding the neurophysiological basis of language processing.

\section{Acknowledgments}

This work was supported by the Austrian Science Foundation ("Herta Firnberg"-project T127) and the German Science Foundation (SFB 360). We wish to express special thanks to Peter Rappelsberger and Baerbel Schack without whom this work would not have been possible. Further we wish to thank Helmuth Petsche, Gert Rickheit, Lorenz Sichelschmidt, Jonathan W. King, and Marta Kutas for helpful discussions and support. The research summarized here would also not have been possible without the technical support of Oliver Filz and Anna Schneider and the help of Werner Chromecek, Carla Berghoff, Kerstin Hadelich, and Alexandra Schuth during data analysis. Many thanks also to Laurie Stowe and an anonymous reviewer for very helpful comments on a previous version of this paper.

\section{References}

Abeles, M., Vaadia, E., Bergman, H., Prut, Y., Haalman, I., \& Slovin, H. (1993). Dynamics of neuronal interactions in the frontal cortex of behaving monkeys. Concepts in Neuroscience, 4, 131-158.

Basar, E. (1998). Brain function and oscillations II: Integrative brain function. Neurophysiology and cognitive processes. Berlin: Springer.

Bastiaansen, M. C. M., van Berkum, J. J. A., \& Hagoort, P. (2002). Event-related $\theta$ power increases in the human EEG during online sentence processing. Neuroscience Letters, 323, 13-16.
Beaumont, J. G., Mayes, A. R., \& Rugg, M. D. (1978). Asymmetry in EEG $\alpha$ coherence and power: Effects of task and sex. Electroencephalography and Clinical Neurophysiology, 45, 393-401.

Binder, J. R. (1997). Neuroanatomy of language processing studied with functional MRI. Clinical Neuroscience, 4, 87-94.

Bressler, S. L., \& Kelso, J. A. S. (2001). Cortical coordination dynamics and cognition. Trends in Cognitive Sciences, 5, 2636.

Brown, C. M., \& Hagoort, P. (Eds.). (1999). The neurocognition of language. New York: Oxford University Press.

Caplan, D., \& Waters, G. S. (1999). Verbal working memory and sentence comprehension. Behavioral and Brain Sciences, 22, 77126

Caramazza, A., \& Hillis, A. E. (1991). Lexical organization of nouns and verbs in the brain. Nature, 349, 788-790.

Challis, R. E., \& Kitney, R. I. (1991). Biomedical signal processing (in four parts). Part 3: The power spectrum and coherence function. Medical \& Biological Engineering \& Computing, 29, 225-241.

Damasio, A. R., \& Geschwind, N. (1984). The neural basis of language. Annual Review of Neuroscience, 7, 127-147.

Damasio, H., Grabowski, T. J., Tranel, D., Hichwa, R. D., \& Damasio, A. R. (1996). A neural basis for lexical retrieval. Nature, 380, 499-505.

Eckhorn, R., Bauer, R., Jordan, W., Brosch, M., Kruse, W., Munk, M., \& Reitboeck, H. J. (1988). Coherent oscillations: A mechanism for feature linking in the visual cortex? Biological Cybernetics, 60, 121-130.

Eggermont, J. J. (1992). Neural interaction in cat primary auditory cortex: Dependence on recording depth, electrode separation, and age. Journal of Neurophysiology, 68, 1216-1228.

Essl, M., \& Rappelsberger, P. (1998). EEG coherence and reference signals: Experimental results and mathematical explanations. Medical \& Biological Engineering \& Computing, 36, 399-406.

Farah, M. J. (1989). The neural basis of mental imagery. Trends in Neuroscience, 12, 395-399.

Finger, S. (1994). Origins of Neuroscience. New York: Oxford University Press.

French, C., \& Beaumont, J. G. (1984). A critical review of EEG coherence studies of hemispheric function. International Journal of Psychophysiology, 1, 241-254.

Gray, C. M., Koenig, P., Engel, A. K., \& Singer, W. (1989). Oszillatory responses in cat visual cortex exhibit inter-columnar synchronization which reflects global stimulus properties. Nature, 338, 334 337.

Harmony, T. (1999). Do specific EEG frequencies indicate different processes during mental calculation? Neuroscience Letters, 266, 2528.

Just, M. A., Carpenter, P. A., Keller, T. A., Eddy, W. F., \& Thulborn, K. R. (1996). Brain activation modulated by sentence comprehension. Science, 274, 114-116.

Kellenbach, M. L., Wijers, A. A., Hovius, M., Mulder, J., \& Mulder, G. (2002). Neural differentiation of lexico-syntactic categories or semantic features? Event-related potential evidence for both. Journal of Cognitive Neuroscience, 14, 561-577.

Kiehl, K. A., Liddle, P. P., Smith, A. M., Mendreck, A., Forster, B. B., \& Hare, R. D. (1999). Neural pathways involved in the processing of concrete and abstract words. Human Brain Mapping, 7, 225233.

King, J. W., \& Just, M. A. (1991). Individual differences in syntactic processing. The role of working memory. Journal of Memory and Language, 30, 580-602.

King, J. W., \& Kutas, M. (1995). Who did what and when? Using word- and clause-level ERPs to monitor working memory usage in reading. Journal of Cognitive Neuroscience, 7, 376-395.

Klimesch, W. (1999). EEG $\alpha$ and $\theta$ oscillations reflect cognitive and memory performance: A review and analysis. Brain Research Reviews, 29, 169-195. 
Kutas, M. (1997). Views on how the electrical activity that the brain generates reflects the functions of different language structures. Psychophysiology, 34, 383-398.

Kutas, M., \& Hillyard, S. A. (1980). Reading senseless sentences: Brain potentials reflect semantic incongruity. Science, 207, 203-205.

Lachaux, J.-P., Rodriguez, E., Martinerie, J., \& Varela, F. J. (1999). Measuring phase synchrony in brain signals. Human Brain Mapping, 8, 194-208.

Leisman, G., \& Ashkenazi, M. (1980). Aetiological factors in dyslexia: IV. Cerebral hemispheres are functionally equivalent. International Journal of Neuroscience, 11, 157-164.

Leoncani, L., \& Comi, G. (1999). EEG coherence in pathological conditions. Journal of Clinical Neurophysiology, 16, 548-555.

Marosi, E., Harmony, T., Becker, J., Reyes, A., Bernal, J., Fernandez, T., Rodriguez, M., Silva, J., \& Guerrero, V. (1995). Electroencephalographic coherences discriminate between children with different pedagogical evaluation. International Journal of Psychophysiology, 19, 23-32.

Mesulam, M. M. (1998). From sensation to cognition. Brain, 121, 1013-1052.

Mueller, H. M., King, J. W., \& Kutas, M. (1997a). Event-related potentials elicited by spoken relative clauses. Cognitive Brain Research, 5, 193-203.

Mueller, H. M., \& Kutas, M. (1996). What's in a name? Electrophysiological differences between spoken nouns, proper names, and one's own name. Neuroreport, 8, 221-225.

Mueller, H. M., Weiss, S., \& Rappelsberger, P. (1997b). EEG coherence analysis of auditory sentence processing. In $\mathrm{H}$. Witte, U. Zwiener, B. Schack, \& A. Doering (Eds.), Quantitative and topological EEG and MEG analysis (pp. 429-431). Jena: Universitaetsverlag.

Murthy, V. N., \& Fetz, E. E. (1992). Coherent 25- to 35-Hz oscillations in the sensorimotor cortex of awake behaving monkeys. Proceedings of the National Academy of Sciences USA, 89, 56705674.

Nagarajan, S., Mahncke, H., Salz, T., Tallal, P., Roberts, T., \& Merzenich, M. M. (1999). Cortical auditory signal processing in poor readers. Proceedings of the National Academy of Sciences USA, 96, 6483-6488.

Nunez, P. L., Srinivasan, R., Westdorp, A. F., Wijesinghe, R. S., Tucker, D. M., Silberstein, R. B., \& Cadush, P. J. (1997). EEG coherency I: Statistics, reference electrode, volume conduction, Laplacians, cortical imaging, and interpretation at multiple scales. Electroencephalography and Clinical Neurophysiology, 103, 499515.

Petsche, H. (1996). Approaches to verbal, visual and musical creativity by EEG coherence analysis. International Journal of Psychophysiology, 24, 145-159.

Petsche, H., \& Etlinger, S. C. (1998). EEG and thinking. Wien: Oesterreichische Akademie der Wissenschaften.

Petsche, H., Etlinger, S. C., \& Filz, O. (1993). Brain electrical mechanisms of bilingual speech management: An initial investigation. Electroencephalography and Clinical Neurophysiology, 86, 385-394.

Pulvermueller, F., Haerle, M., \& Hummel, F. (2001). Walking or talking? Behavioral and neurophysiological correlates of action verb processing. Brain and Language, 78, 134-168.

Pulvermueller, F., Preissl, H., Lutzenberger, W., \& Birbaumer, N. (1996). Brain rhythms of language: Nouns versus verbs. European Journal of Neuroscience, 8, 937-941.

Rappelsberger, P. (1998). Probability mapping of power and coherence: Technical aspects. In H. Petsche, \& S. Etlinger (Eds.), EEG and thinking (pp. 63-78). Wien: Oesterreichische Akademie der Wissenschaften.

Rappelsberger, P., \& Petsche, H. (1988). Probability mapping: Power and coherence analyses of cognitive processes. Brain Topography, $1,46-54$.
Rappelsberger, P., Weiss, S., \& Schack, B. (1999). Coherence and phase relations between EEG traces recorded from different locations. In R. Miller (Ed.), Time and the brain (pp. 297-330). Amsterdam: Harwood Academic Publishers.

Roelfsema, P. R., Engel, A. K., Koenig, P., \& Singer, W. (1997). Visuomotor integration is associated with zero time-lag synchronization among cortical areas. Nature, 385, 157-161.

Rugg, M. D., \& Venables, P. H. (1980). EEG correlates of the acquisition of high- and low-imagery words. Neuroscience Letters, 16, 67-70.

Sarnthein, J., Petsche, H., Rappelsberger, P., Shaw, G. L., \& von Stein, A. (1998). Synchronization between prefrontal and posterior association cortex during human working memory. Proceedings of the National Academy of Sciences USA, 95, 7092-7096.

Schack, B., Chen, A. C., Mescha, S., \& Witte, H. (1999a). Instantaneous EEG coherence analysis during the Stroop task. Clinical Neurophysiology, 110, 1410-1426.

Schack, B., Grieszbach, G., Arnold, M., \& Bolten, J. (1995). Dynamic cross-spectral analysis of biological signals by means of bivariate ARMA processes with time-dependent coefficients. Medical \& Biological Engineering \& Computing, 33, 605-610.

Schack, B., \& Krause, W. (1995). Dynamic power and coherence analysis of ultra short-term cognitive processes - A methodical study. Brain Topography, 8, 1-10.

Schack, B., Rappelsberger, P., Anders, C., Weiss, S., \& Moeller, E. (2000). Quantisation of synchronisation processes by coherence and phase and its application in analysis of electrophysiological signals. International Journal of Bifurcation and Chaos, 11, 2565-2586.

Schack, B., Rappelsberger, P., Vath, N., Weiss, S., Moeller, E., \& Grieszbach, G. (2001). EEG frequency and phase coupling during human information processing. Methods of Information in Medicine, 40, 106-111.

Schack, B., Rappelsberger, P., Weiss, S., \& Moeller, E. (1999b). Adaptive phase estimation and its application in EEG analysis of word processing. Journal of Neuroscience Methods, 93, 49-59.

Schack, B., Vath, N., Petsche, H., Geissler, H.-G., \& Moeller, E. (2002). Phase-coupling of $\theta-\gamma$ EEG rhythms during short-term memory processing. International Journal of Psychophysiology, 44, 143-163.

Schack, B., Weiss, S., \& Rappelsberger, P. (1996). Dynamic topographic methods of coherence analysis of cognitive processes. Medical \& Biological Engineering \& Computing, 34, 207208.

Schack, B., Weiss, S., \& Rappelsberger, P. (2003). Cerebral information transfer during word processing: Where and when does it occur and how fast is it. Human Brain Mapping, 19, 18-36.

Semenza, C., \& Zettin, M. (1989). Evidence from aphasia for the role of proper names as pure referring expressions. Nature, 342, 678-679.

Shaw, J. C. (1984). Correlation and coherence analysis of the EEG: A selective tutorial review. International Journal of Psychophysiology, $1,255-266$.

Sheppard, W. D., II, \& Boyer, R. W. (1990). Pretrial EEG coherence as a predictor of semantic priming effects. Brain and Language, 39, 57-68.

Shiota, M., Koeda, T., \& Takeshita, K. (2000). Cognitive and neurophysiological evaluation of Japanese dyslexia. Brain and Development, 22, 421-426.

Singer, W., \& Gray, C. M. (1995). Visual feature integration and the temporal correlation hypothesis. Annual Review of Neuroscience, $18,555-586$

Sklar, B., Hanley, J., \& Simmons, W. W. (1972). An EEG experiment aimed toward identifying dyslexic children. Nature, 240, 414 416.

Towle, V. L., Carder, R. K., Khorasani, L., \& Lindberg, D. (1999). Electrocorticographic coherence patterns. Journal of Clinical Neurophysiology, 16, 528-547.

Tucker, D. M., Dawson, S. L., Roth, D. L., \& Penland, J. G. (1985). Regional changes in EEG power and coherence during cognition: 
Intensive study of two individuals. Behavioral Neuroscience, 99, 564-577.

Varela, F., Lachaux, J.-P., Rodriguez, E., \& Martinierie, J. (2001). The brainweb: Phase synchronization and large-scale integration. Nature Reviews Neuroscience, 2, 229-239.

Volf, N. V., \& Razumnikova, O. M. (1999). Sex differences in EEG coherence during a verbal memory task in normal adults. International Journal of Psychophysiology, 34, 113-122.

von Stein, A., Rappelsberger, P., Sarnthein, J., \& Petsche, H. (1999). Synchronization between temporal and parietal cortex during multimodal object processing in man. Cerebral Cortex, 9, $137-150$.

Walter, D. O. (1968). Coherence as a measure of relationship between EEG records. Electroencephalography and Clinical Neurophysiology, 24, 282.

Warburton, E., Wise, R. J. S., Price, C. J., Weiller, C., Hadar, U., Ramsay, S., \& Frackowiak, R. S. J. (1996). Noun and verb retrieval by normal subjects: Studies with PET. Brain, 119, 159-179.

Warrington, E. K., \& Shallice, T. (1984). Category specific semantic impairments. Brain, 107, 829-854.

Weiss, S., Berghoff, C., Rappelsberger, P., \& Mueller, H. M. (2001a). Elektrophysiologische Hinweise zur Kategorisierung von Verben. Proceedings of the 1. Jahrestagung der Gesellschaft fuer Aphasieforschung und Behandlung: Bielefeld, p. 64.

Weiss, S., Cromecek, W., \& Rappelsberger, P. (1998b). Elektrophysiological signs for a differentiation between good and poor memory performers. European Journal of Neuroscience (Suppl.10), 142.

Weiss, S., Mueller, H. M., King, J. W., Kutas, M., \& Rappelsberger, P. (2001b). EEG-coherence analysis of naturally spoken English relative clauses. Brain Topography, 13, 317.
Weiss, S., Mueller, H. M., \& Rappelsberger, P. (1998a). EEGcoherence analysis of spoken names and nouns processing. Society for Neuroscience, 24, 462.9 .

Weiss, S., Mueller, H. M., \& Rappelsberger, P. (1999). Processing concepts and scenarios: Electrophysiological findings on language representation. In A. Riegler, M. Peschl, \& A. v. Stein (Eds.), Understanding representation in the cognitive sciences (pp. 237-246). New York: Plenum Press.

Weiss, S., Mueller, H. M., \& Rappelsberger, P. (2000). Theta synchronisation predicts efficient memory encoding of concrete and abstract nouns. Neuroreport, 11, 2357-2361.

Weiss, S., \& Rappelsberger, P. (1996). EEG coherences within the 13$18 \mathrm{~Hz}$ band as correlates of a distinct lexical organization of concrete and abstract nouns in humans. Neuroscience Letters, 209, $17-20$.

Weiss, S., \& Rappelsberger, P. (1998). Left frontal EEG coherence reflects modality independent language processes. Brain Topography, 11, 33-42.

Weiss, S., \& Rappelsberger, P. (2000). Long-range EEG synchronization during word encoding correlates with successful memory performance. Cognitive Brain Research, 9, 299-312.

Weiss, S., Rappelsberger, P., Schack, B., \& Mueller, H. M. (2003). Kohärenz- und Phasenuntersuchungen und ihre Bedeutung für die Untersuchung von Sprachprozessen. In H. M. Mueller, \& G. Rickheit (Eds.), Neurokognition der Sprache (pp. 211-258). Tübingen: Stauffenburg.

West, W. C., \& Holcomb, P. J. (2000). Imaginal, semantic, and surface-sevel processing of concrete and abstract words: An electrophysiological investigation. Journal of Cognitive Neuroscience, 12, 1024-1037. 